\title{
Skin and Other Reactions to Radiotherapy - Clinical Presentation and Radiobiology of Skin Reactions
}

\author{
Wolfgang Dörr ${ }^{\mathrm{a}, \mathrm{b}}$ \\ ${ }^{a}$ Klinik und Poliklinik für Strahlentherapie und Radioonkologie, \\ ${ }^{b}$ Experimentelles Zentrum, Medizinische Fakultät Carl Gustav Carus, \\ Technische Universität Dresden, Dresden, Deutschland
}

The skin represented the dose-limiting organ in radiotherapy over long periods of time. In the first third of the 20th century, radiotherapy was associated with deposition of significant radiation doses in the superficial compartments of the skin. Therefore, all major radiobiological principles, such as effects of fractionation on radiation sensitivity or volume/area effects, were initially based on observations in epidermal radiation reactions.

The development of radiation sources producing mega-voltage X-rays resulted in translocation of dose maxima into the subcutaneous soft tissue. With this, and with the introduction of multiple-field irradiation techniques, severe radiation effects in the skin were almost completely prevented. However, skin reactions are still relevant to critical skin areas, such as intertriginous regions. Also, the treatment of skin tumours, which requires high skin doses, is associated with substantial skin effects. Combinations of radiotherapy, e.g. with chemotherapy or UV exposure, can significantly aggravate skin effects. Moreover, accidental radiation exposure is frequently associated with significant skin doses. Therefore, early and late reactions of the skin must still be considered clinically relevant.

\section{Pathophysiology and Clinical Presentation of Skin Reactions}

Radiation effects in skin represent an orchestrated response of all individual tissue components, i.e. epidermis, hair follicles, vasculature, glands, 


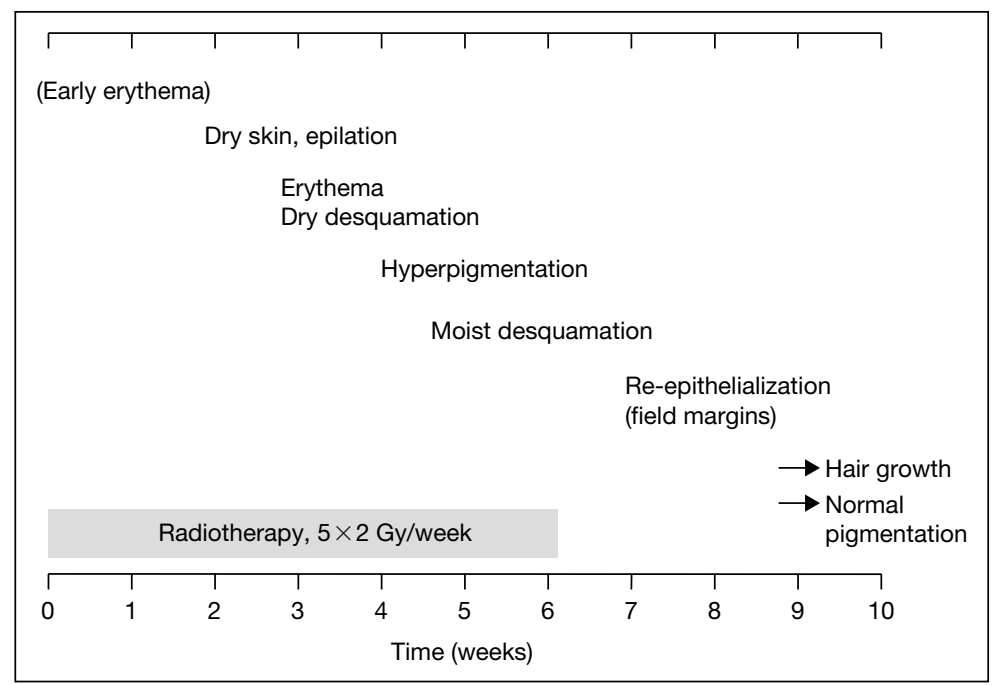

Fig. 1. Time course of early skin reactions induced by conventional radiotherapy with $5 \times 2 \mathrm{~Gy} /$ week over 6 weeks, if the same skin area is exposed to the maximum dose of $2 \mathrm{~Gy}$ at each dose fraction. Overall treatment time is indicated on the abscissa.

melanocytes and connective tissue (fibroblasts), to radiation exposure [Dörr et al., 2005; Fajardo et al., 2001; Trott and Kummermehr, 1991; Trott et al., 2002].

The time course of the early skin response during conventional radiotherapy with $5 \times 2$ Gy per week, based on the assumption that the same skin area receives the maximum dose of $2 \mathrm{~Gy}$ at each fraction, is illustrated in figure 1. Early erythema, which is frequently observed after accidental exposure with high single doses, is rarely seen during radiotherapy. Initial skin effects are observed in the second to third treatment week. Irradiation of the sebaceous and sweat glands results in dry skin. The vascular response to radiation exposure, including synthesis and release of vasoactive substances, yields erythema, oedema and perivascular infiltration of inflammatory cells. First symptoms of epilation, as a sign of radiation injury to hair follicles, can be found from weeks 2 to 3 of conventional fractionation.

Subsequently, the radiation effect is dominated by the epidermal reaction. Proliferative impairment of epidermal basal cells results in insufficient cellular supply to the spinous cell layer. The consequence is epithelial atrophy, which clinically presents as dry desquamation, radiodermatitis sicca, that can progress to more severe changes, i.e. moist desquamation. Most severe reactions are necrosis and haemorrhage, as a combination of epidermal changes and damage 
to the capillary endothelial cells [Fajardo et al., 2001]. Promoted by surviving cells from within the treatment area and/or migrating cells from the margins of the treatment field, healing can occur from each of the reaction grades. Usually, recovery from early symptoms is complete. Pigment changes in the regenerating hair follicles can result in changes in hair colour in the irradiated areas. Clinical examples of early radiation effects in skin are illustrated in Seegenschmiedt et al. [2000].

After latencies of months to years, late epidermal atrophy develops due to insufficient supply with nutrients following a reduction in the number of capillaries of the irradiated vasculature [Fajardo et al. 2001]. Moreover, parakeratotic, hyperproliferative epidermal reactions can be observed. Late vascular effects, besides a loss of capillaries, frequently manifest as telangiectasia [Trott and Kummermehr, 1991; Trott et al., 2002]. Dermal fibrosis is the predominant manifestation of radiation effects in soft tissue fibroblasts. Mitotic fibroblasts differentiate into post-mitotic fibrocytes. These produce a significantly higher amount of collagen, which is deposited in the tissue.

Consequential late effects, i.e. late radiation sequelae affected by the extent of the early reaction, i.e. the combination of severity and duration, are seen as a consequence of the impaired barrier function associated with early epidermal changes [Dörr and Hendry, 2001]. This can cause additional mechanical and chemical trauma to soft tissue and vessels. Consequential late effects substantially contribute to late skin reactions seen in clinical routine.

\section{Times to Clinical Manifestation}

Times to clinical manifestation of early (epidermal) radiation effects are over a wide range - independent of the radiation dose and are related to the normal epithelial turnover times. Physiological cell loss, predominantly caused by mechanical shedding at the surface, is independent of the radiation treatment and continues at a near normal rate. Hence, cell numbers decrease continuously due to lacking cellular supply, resulting in clinical effects if a threshold is reached. In contrast, the time to clinical healing of the effects as well as to complete restoration of cell numbers is based on the (dose-dependent) number of surviving cells, and hence depends on the radiation dose [Dörr and Herrmann, 2003].

In contrast to early effects, times to chronic clinical effects are the shorter the higher the dose was. This is based on higher rates of capillary loss and fibroblast differentiation. This results in earlier manifestation of the clinical response. Late radiation effects are usually irreversible and progressive in nature, with a dose-dependent progression rate. Therefore, the tolerance doses 
for late effects clearly decrease with an increase in follow-up time [Dörr et al., 2005] if clinically relevant doses are administered.

\section{Radiation Tolerance}

The doses required to induce early skin reactions are in the range of $30-40 \mathrm{~Gy}$, given in 2-Gy fractions. Moist reactions develop from 50-60 Gy [Trott and Kummermehr 1991]. Higher doses can result in permanent non-healing reactions. Permanent epilation is seen in $50 \%$ of the patients after doses in the range of $40 \mathrm{~Gy}$ given in 2-Gy fractions [Lawenda et al., 2004]; the tolerance dose for permanent epilation is in the range of $10 \mathrm{~Gy}$. Irreversible effects in sweat and sebaceous glands, e.g. lacking axillary transpiration, develop at doses above 50 Gy.

Moreover, the clinical radiation tolerance of the skin varies with localization. While skin folds, the skin of the throat and of the flexion areas of joints are particularly sensitive, mainly due to additional mechanical stress, the skin of the head, neck, face, palms and soles, is relatively insensitive. Additional trauma, including chemotherapy, can significantly aggravate the radiation effects in the skin [Dörr et al., 2005].

\section{Wound Healing and Radiotherapy}

Wound healing in the irradiated area is a particular problem. Post-operative radiotherapy should not start before removal of the suture material, i.e. after an interval of about 2 weeks, to avoid impairment of wound healing [Trott et al., 2002].

Surgical treatment of the skin in irradiated areas shortly after radiotherapy is usually compromised by hyperaemia and intense perfusion. However, effects on wound healing are scarce if an interval of 4-6 weeks is allowed between treatments. For surgery months to years after radiotherapy, the chronic tissue changes described above, have to be considered, and may result in significant impairment of wound healing [Trott et al., 2002].

\section{Radiobiology of the Skin}

The main radiobiological parameters of the early epidermal response to radiation are the overall treatment time and the irradiated area. With increasing time of radiotherapy, the radiation tolerance significantly increases following a 
process depicted as repopulation, i.e. the regeneration response of the tissue that is stimulated by the progression of radiation-induced changes, mainly continuing epithelial cell loss [Dörr and Herrmann, 2003]. Repopulation is based on increased proliferation rates of surviving cells, in combination with a restructuring of the proliferative organization. Moreover, residual proliferative activity of the radiation-damaged cells significantly contributes to overall cell production. The underlying mechanisms were summarized as the ' 3 As' of radiation-induced repopulation [Dörr, 1997, 2003].

Epidermal restoration depends on the number of surviving (stem) cells, which have a high migration potential. This is the basis of the substantial area effect observed in skin. With smaller areas $\left(<4-5 \mathrm{~cm}^{2}\right)$ a marked increase in radiation tolerance is seen with decreasing field size. In contrast, skin tolerance to larger treatment fields is independent of the irradiated area. In the orthovoltage radiotherapy era, this has been exploited in 'grid' irradiation techniques [Dörr et al., 2005]. Multiple small treatment areas, separated by $1-\mathrm{cm}^{2}$ areas that received no irradiation or a lower dose, allowed administration of large single doses (20-30 Gy) without severe acute effects. However, intense formation of telangiectasia was regularly observed in the irradiated areas [Dörr et al., 2005].

For late effects in skin, the size of the radiation dose per fraction plays a major role, and hence hyperfractionation can be applied to reduce late sequelae. However, a reduction in acute effects, by avoidance of additional trauma, or - as currently tested in preclinical studies, by administration of growth factors - can significantly decrease the consequential component of the late response.

\section{References}

Dörr W: Three A's of repopulation during fractionated irradiation in squamous epithelia: asymmetry loss, acceleration of stem-cell divisions and abortive divisions. Int J Radiat Biol 1997;72:635-643.

Dörr W: Modulation of repopulation processes in oral mucosa: experimental results. Int J Radiat Biol 2003;79:531-537.

Dörr W, Hendry JH: Consequential late effects in normal tissues. Radiother Oncol 2001;61:223-231.

Dörr W, Herrmann TH: Akute Strahlenveränderungen der Gewebe; in Bamberg M, Molls M, Sack H (eds): Radioonkologie. Grundlagen. Germering/Munich, Zuckschwerdt, 2003, vol 1, pp 244-250.

Dörr W, Herrmann Th, Riesenbeck D: Prävention und Therapie von Nebenwirkungen in der Strahlentherapie. Bremen, UNI-MED Science, 2005.

Fajardo LFLG, Berthrong M, Armstrong RE: Radiation Pathology. New York, Oxford University Press, 2001.

Lawenda BD, Gagne HM, Gierga DP, Niemierko A, Wong WM, Tarbell NJ, Chen GT, Hochberg FH, Loeffler JS: Permanent alopecia after cranial irradiation: Dose-response relationship. Int J Radiat Oncol Biol Phys 2004;60:879-887.

Seegenschmiedt MH: Akute Nebenwirkungen an der Haut bei Strahlentherapie. Bildanhang; in Dörr W, Zimmermann JS, Seegenschmiedt MH (eds): Nebenwirkungen in der Radioonkologie. Munich, Urban \& Vogel, 2000, pp 287-294. 
Trott K-R, Herrmann TH, Dörr W: Strahlenwirkungen auf Normalgewebe. Munich, Urban \& Vogel, 2002.

Trott KR, Kummermehr J: Radiation effects in skin; in Scherer E, Streffer C, Trott KR (eds): Radiopathology of Organs and Tissues. Berlin, Springer, 1991, pp 33-66.

Prof. Dr. Wolfgang Dörr

Klinik und Poliklinik für Strahlentherapie und Radioonkologie

Medizinische Fakultät Carl Gustav Carus

Fetscherstrasse 74

DE-01307 Dresden (Germany)

Tel. +49 351458 3390, Fax +49 351458 4347, E-Mail doerr@rcs.urz.tu-dresden.de 